\title{
A ROLE FOR OXIDATIVE STRESS IN EXFOLIATION SYNDROME
}

\author{
D. Kovachev', G. Koliakos ${ }^{3}$, T. Mitov², R. Kovachev², D. Mitova ${ }^{2}$ \\ ${ }^{1}$ Medical University-Varna, Dept. of Biology, ${ }^{2}$ Medical eye center "St. Petka"- Varna \\ ${ }^{3}$ Aristotle University of Thessaloniki, Medical School, Dept. of Biochemistry, Thessaloniki, Greece
}

Reviewed by: Assoc. prof. E. Kontrova, PhD

\begin{abstract}
Exfoliation syndrome (XFS) is the most common identifiable condition leading to the development of open angle glaucoma. During cataract surgery aqueous humour samples were obtained from $\mathbf{2 5}$ consecutive eyes of 25 patients with XFS and an equal number of controls. We investigated the concentration of Endothelin -1 (ET-1) in the aqueous humour with and without XFS. The activity of growth factor was referred to as a percentage of the effect of foetal calf serum. Ascorbic acid concentration, a major antioxidant in the eye, was assessed with a microplate assay method. The increased concentrations of ET-1 and growth factor, and the depression of ascorbic acid in the aqueous humour of XFS patients provide evidence suggesting the possible role of the oxidative stress in the pathogenesis of XFS.
\end{abstract}

Keywords: exfoliation syndrome, glaucoma, oxidative stress.

\section{INTRODUCTION}

Exfoliation syndrome (XFS) is an age-related extracellular matrix disorder, which often leads to the development of exfoliative glaucoma (XFG), with worse prognosis than primary open-angle glaucoma (1). The main characteristic of XFS is the progressive accumulation of a fibrillar extracellular material (exfoliation material) in many ocular and systemic tissues. The precise pathogenetic mechanism responsible for the development of XFS and its progression to XFG remain, as yet, obscure (2).

Recent aqueous studies have provided evidence that oxidative stress may play a major role in the pathogenesis of XFS. The aqueous level of 8-isoprostaglandine F2a (8-IsoPGF2a), a marker of oxidative stress, was found to be 5 times greater in XFS (3). Recently, matrix metalloproteinase 2 (MMP 2) was reported to be elevated in the aqueous of XFS and XFG patients. It has been established that 8-IsoPGF2a promotes the secretion of endothelin-1 (ET-1), and MMP 2 promotes the conversion of ET-1 from its precursor protein, big endothelin (4).

ET-1 is a 21 aminoacid peptide with mitogenic properties, which is considered to be the most potent vasoconstrictor in the body (5). In addition, ET-1 constricts the human trabecular meshwork, and to a lesser degree the ciliary muscle. ET-1 is actively secreted by human non-pigmented ciliary epithelial cells to the aqueous humour and this leads to a 3-4 times higher level of endothelin like immunoreactivity in the aqueous humour than that found in

Address for correspondence:

D. Kovachev, Dept. of Biology, Medical University Prof. Dr. Paraskev Stoyanov, 55 Marin Drinov St., BG-9002, Varna, BULGARI the plasma of healthy subjects (6). Endothelin like immunoreactivity has been found higher in the aqueous humour of patients with primary open-angle glaucoma (POAG) and cataract compared to cataract controls (7), which implies a role for ET in intraocular pressure regulation in humans.

To date the level of ET-1 in the aqueous humour of patients with XFS, or XFG, has not been evaluated. This measurement may provide a valuable insight into the role of ET-1 in this condition and may provide a logical explanation for a number of vascular alterations in XFS.

Ascorbic acid (Vit.C) acts as an electron donor in several reactions that require reducing equivalents. The oxidized form of vit.C is the dehydroascorbic acid. Increasing evidence suggests that within the aqueous humour ascorbic acid plays essential role in the antioxidant defence mechanism of the eye protecting ocular tissues against photooxidative damage by acting as a free radical scavenger (8).

Since growth factors and proteases can be activated by free radicals, their involvement in XFS pathobiology requires further elucidation. Previous studies have indicated that protein concentration is increased in the anterior chamber in XFS (9). This can be attributed to alterations of the integrity of the blood-aqueous barrier (BAB) (10). This breakdown may disrupt the balance among growth factors in the aqueous humour.

Growth factors are molecules that induce cellular proliferation. A variety of growth factors have been isolated and characterized from biological fluids by measuring their effect on DNA synthesis using $(3 \mathrm{H})$ thymidine incorporation assay in cell cultures $(11,12)$. However, the functional role of these molecules extends beyond mitogenicity. Growth 
factors may induce cellular migration, stimulate extracellular matrix synthesis and control angiogenesis. Growth factors are present in the aqueous and their activity may play an important role in anterior chamber pathology (13).

We therefore, investigated the aqueous humour ET-1 concentration, growth factor(s), ascorbic acid and protein concentration in age-matched cataract patients with and without XFS.

\section{PATIENTS AND METHODS}

Aqueous humour samples were collected at the Department of Ophthalmology, Medical University of Varna, Bulgaria and the University Department of Ophthalmology, Aristotle University, Thessaloniki, Greece. An informed consent was obtained from all patients. We entered in this prospective study consecutive aqueous samples from patients with and without XFS who exhibited normal intraocular pressure and were undergoing routine cataract surgery. Excluded were patients with other ophthalmic diseases (e.g. glaucoma, uveitis, progressive retinal disease etc) and systemic diseases (e.g. diabetes, arthritis) that might have influenced the levels of the parameters under investigation.

All patients underwent a comprehensive ocular examination prior to enrolment. A slit-lamp examination was performed, after pupillary dilatation, the day prior to phacoemulcification surgery to document the presence of signs consistent with XFS. All patients included had an IOP consistently below $20 \mathrm{~mm} \mathrm{Hg}$, and did not receive any local medication except artificial tears, or standard mydriatics prior to cataract surgery. For the purposes of this study only patients exhibiting typical exfoliation material deposits on the lens and/or pupillary border were deemed to have XFS. Patients exhibiting pigment liberation signs commensurate with the definition of exfoliation suspect (14) were excluded from the study to ensure lack of possible contamination of the control group. An attempt was made to keep all conditions uniform prior to aqueous acquisition (e.g. duration of body rest in supine position in the operating theatre and temperature of the operating theatre). A small amount of humour (15-40 ?1) was aspirated through an ab-externo limbal paracentesis site using a 27 -gauge needle on a tuberculin syringe, with special care to avoid blood contamination. Immediately after collection, aqueous samples were stored at $-70 \mathrm{o} C$ until biochemical analysis. This investigation included a total of 50 consecutive aqueous samples, 25 from cataract patients with XFS (mean age 75.1 6.9 years) and 25 from age-matched cataract patients without XFS (mean age 74.37 .1 years).

The aqueous concentration of ET-1 as estimated with a commercial immunoassay kit (Biomedica $\mathrm{GmbH}$, Wien, Austria). The kit is a sandwich type enzyme linked immunoassay designed to determine endothelin (1-21) directly in biological fluids. The kit uses a 96 well plate precoated with a polyclonal antibody and a highly specific monoclonal detection antibody. It possesses a $100 \%$ crossreactivity for ET-1 and ET-2, $<5 \%$ for ET-3 and $<1 \%$ for big Endothelin 1, or 2. A calibration curve was constructed using the standard ET-1 solutions provided by the manufacturer with six standard solutions within a range of 0,16 to $0,8 \mathrm{pg} / \mathrm{ml}$. For the purposes of this study this kit assessed virtually only ET-1 since the kit uses ET-1 standards and the presence and biological effect of ET-2 in ocular tissues is considered negligible (15). Ten ?1 of aqueous samples diluted with 190 ? 1 of the assay buffer, provided by the manufacturer, were added to the appropriate wells. The ET-1 concentration measurement assay protocol was then followed according to the instructions of the manufacturer. The lower detection limit of the assay was determined as the concentration of ET-1 measured at three standard deviations from zero along the calibration curve and was calculated to be $0,13 \mathrm{pg} / \mathrm{ml}$. Intra-assay variation was found to be $7,3 \%$ for a control concentration of about $8 \mathrm{pg} / \mathrm{ml}$ measured 6 times.

For the evaluation of ascorbic acid concentration, a microplate assay method was used in accordance with the technique described by Shichi et al.(16). The aqueous humour samples (20 1 each) were treated with $10118 \%$ trichloroacetic acid, incubated at room temperature for 15 min and centrifuged for $15 \mathrm{~min}$ at $12,000 \mathrm{~g}$ to precipitate protein. Protein was then measured in the sediment using the bicinchonic acid (BCA) assay as described before (9). Ten 1 of each supernatant, standard solution or control was mixed in a microplate well with 51 of $1 \mathrm{M} \mathrm{NaOH}$ and was incubated for $30 \mathrm{~min}$ at room temperature. After incubation another 101 of each supernatant was placed in a second well. Each sample was then mixed with 2001 of BCA reagent (Pierce, USA). After incubation for $30 \mathrm{~min}$ at room temperature the absorbance at $562 \mathrm{~nm}$ was measured. Ascorbic acid (Sigma-Aldrich Chemie GmBh, Germany) solutions with known concentrations, treated as described for the samples, served as standards for the calibration curve.

Growth factor activity was estimated using a modification of the $(3 \mathrm{H})$ thymidine incorporation assay adapted for the McCoy cell line (17). Two hundred microlitres of cell suspension $(20,000 \mathrm{cells} / \mathrm{ml})$ in Eagle's medium (EMEM), containing $10 \% \mathrm{FCS}$, was placed in 96-well cell culture plates (Costar). The cells were cultured for 3 days at $37 \mathrm{oC}$ in $5 \% \mathrm{CO} 2$. On the 4 th day the culture medium was replaced with EMEM without FCS. Ten microlitres of each sample and 51 of culture medium containing $1 \mathrm{Ci}$ of 6-(3H)thymidine(Dupont NEN - NET 335-20, 1 Ci/mmol) was then added to each cell culture well. After $19 \mathrm{~h}$ the medium was aspirated. The fixed cells were washed and lysed with $5010.25 \mathrm{M} \mathrm{NaOH}$. The lysates were counted in a liquid scintillation counter (1219 Rackbeta LKB). The results of the assay were reported as percentage of $(3 \mathrm{H})$ thymidine incorporation in comparison to controls that contained $10 \%$ FCS.

For statistical calculations the GraphPad InStat statistical package for Windows was used (version 3.00 for Windows 96, GraphPad Software, San Diego, USA). Normal distri- 
bution fitting was checked by the Kolmogorov-Smirnov goodness of the fit test. Statistical significance of the differences between XFS and controls was calculated by the two-tailed unpaired Student $t$ test with Welch correction. Correlations between sets of data were estimated by Pearson correlation.

\section{RESULTS AND DISCUSSION}

Ascorbic acid was measured in all samples collected. The mean concentration of Vit.C in the aqueous humour from patients with XFS ( $0.870 .38 \mathrm{mM}$, range 0.18 to $1.70 \mathrm{mM})$ was found to be significantly lower than that from age-matched control patients $(1.160 .49 \mathrm{mM}$, range 0.43 to $3.1 \mathrm{mM} ; \mathrm{P}=0.0065)($ Table 1$)$. Our data are similar to previously reported values (18). In the present study the concentration of ascorbic acid in the aqueous humour was estimated using a microplate assay. This technique offers the advantage of simultaneous measurement of multiple XFS aqueous samples and controls in the same assay, enabling a more reliable comparison between the two groups. However, this method measures the reducing capacity of Vit.C and not include dehydroascorbic asid. The decrease in Vit.C levels in aqueous appears to be related to the XFS because all patients had senile cataract and did not receive any topical medication. Therefore, the most likely etiology for the reduced ascorbic levels in XFS is either depletion of Vit.C induced by increased levels of free radicals or impaired ascorbic transport through the ciliary epithelium. This question cannot be resolved in the present study because the limited amount of aqueous that can be collected during cataract surgery was not sufficient to allow measurement of oxidized ascorbic acid. However, our finding suggests a role of free radicals in the pathogenesis of XFS.

In the present study the human aqueous humour ET-1 concentration was measured, to the best of our knowledge, for the first time employing a specific immunoassay.

Table 1. Mean age and biochemical parameters investigated in the aqueous humour of patients with exfoliation syndrome (XFS) and age-matched controls

\begin{tabular}{||l|c|c|c||}
\hline \hline & $\begin{array}{c}\text { XFS } \\
(\mathbf{n}=\mathbf{2 5})\end{array}$ & $\begin{array}{c}\text { Controls } \\
(\mathbf{n}=\mathbf{2 5})\end{array}$ & $\boldsymbol{P}$ value \\
\hline Mean age & $75.1 \pm 6.9$ years & $74.3 \pm 7.1$ years & 0.302 \\
\hline ET-1 concentration & $4.6 \pm 2.3 \mathrm{pg} / \mathrm{ml}$ & $2.8 \pm 1.71 \mathrm{pg} / \mathrm{ml}$ & 0.003 \\
\hline Total protein & $\begin{array}{c}0.380 \pm 0.159 \\
\mathrm{mg} / \mathrm{ml}\end{array}$ & $\begin{array}{c}0.279 \pm 0.144 \\
\mathrm{mg} / \mathrm{ml}\end{array}$ & 0.023 \\
\hline Ascorbic acid & $0.87 \pm 0.38 \mathrm{mM}$ & $1.16 \pm 0.49 \mathrm{mM}$ & 0.065 \\
\hline Growth factor activity & $35.8 \% \pm 13.9 \%$ & $9.18 \% \pm 6.04 \%$ & 0.00 \\
\hline \hline
\end{tabular}

The values of ET-1 did pass the Kolmogorov-Smirnov normality test for both groups. The mean concentration of ET-1 in the aqueous samples from XFS patients was significantly greater $(4,6 \pm 2,3 \mathrm{pg} / \mathrm{ml}$; range $0,9-9,1 \mathrm{pg} / \mathrm{ml})$ than that measured in the aqueous of age-matched controls ( 2,8 $\pm 1,71 \mathrm{pg} / \mathrm{ml}$; range $0,5-5,9 \mathrm{pg} / \mathrm{ml}) ;(\mathrm{P}=0,003$; Student- $\mathrm{t}$ test)(Table 1).

The values of total protein concentration also passed the Kolmogorov-Smirnov normality test for both groups. The mean total protein concentration in the aqueous humour from patients with XFS $(0,380 \pm 0,159 \mathrm{mg} / \mathrm{ml}$; range $0,106-0,806 \mathrm{mg} / \mathrm{ml}$ ) was significantly greater than that of the age-matched control patients $(0,279 \pm 0,144 \mathrm{mg} / \mathrm{ml}$; range 0,085-0,542 mg/ml), ( $\mathrm{P}=0,023$; Student-t test)(Table 1).

In contrast, no correlation could be established between ET-1 and total protein levels, $(\mathrm{P}=0,73$; Pearson correlation test). Furthermore, ET-1 and protein concentrations did not correlate with age $(\mathrm{P}=0,78$ and $\mathrm{P}=0,578$ respectively; Pearson correlation test). Therefore, the increased protein concentration found in the aqueous of XFS patients in our study cannot be attributed to a disruption of the blood aqueous barrier induced by the increased ET-1 concentration. In addition, increased aqueous ET-1 levels may not be explained by the increased permeability of the blood aqueous barrier in our study. It is also relevant that the plasma concentration of ET-1 in patients with XFG does not differ from that of healthy controls (data not shown). Since ET-1 is considered one of the most powerful vasoconstrictors, the elevated aqueous humour concentration of this molecule may have a significant role in the pathophysiology of the iris vascular changes in XFS. Elevated ET-1 level in the aqueous humour may provide a logical explanation for the well described ischaemic vascular alterations in the anterior segment of patients with XFS. Further research is required to clarify the role of ET-1 in the development of intraocular pressure elevation in XFS, the progression to XFG and the possibility of increased susceptibility to glaucomatous damage in patients with XFG.

A $(3 \mathrm{H})$ thymidine incorporation assay was used to measure growth factor activity in the aqueous of patients with XFS and age-matched controls. In McCoy fibroblast cell culture, XFS samples exhibited a growth factor activity ranging from $10.3 \%$ to $69 \%$ (mean $35.8 \% 13.9 \%$ ), whereas normal controls exhibited activity ranging from $1,3 \%$ to $21.5 \%$ (mean 9.18\% 6.04\%). This difference was significant ( $\mathrm{P}$ $<0.001)($ Table 1). The incorporation assay used estimates cell proliferation, but the biological role of the growth factors involves other cellular functions as well. Increased growth factor activity in aqueous from XFS patients may be attributed either to increased concentration of one of the established growth factors or to the presence of novel once that may not normally be present in the aqueous. Variations in the ratio between existing growth factors and/or activation of latent forms of growth factors may also influence growth factor activity and account the findings of this study. Although our findings indicate that growth factors play a pole in the development of XFS, their significance, composition and function require further elucidation.

The increased concentrations of ET-1 and growth factor, and the depression of ascorbic acid in the aqueous humour of XFS patients provide evidence suggesting its possible role in the pathogenesis of XFS. 
D. Kovachev, G. Koliakos, T. Mitov ...

\section{ACKNOWLEGEMENT}

We acknowledge the operative staff - Dr Fileva, Dr Milkova, Dr Paskov - for excellent work during harvesting the aqueous samples and the excellent technical assistance of Ms Melpomeni Christophoridou.

\section{REFERENCES}

1. Ritch, R, U. Schlotzer-Schrehardt. 2001 Surv.Ophthalmol. 45, 265-315.

2. Ritch, R. 2002 -Acta Ophthalmol. Scand. 80, 465-467.

3. Koliakos, G., A Konstas, U. Schlotzer-Schrehardt, G. Hollo, I. Katsimbris, et al. 2003 - Br.J Ophthalmol. 87,353-356.

4. Schlotzer-Schrehardt, U., J. Lommatzsch, M. Kuchle, A. Konstas. 2003 - Invest. Ophthalmol. Vis. Sci. 44, 1117-1125.

5. Fernandez-Patron, C., M. Radomski, S. Davidge. 1999 - Circ.Res.85, 906-911.

6. Haefliger, I., J. Flammer, J. Beny, t. Luscher. 2001 -Prog. Retin.Eye Res. 20:209-225.
7. Noske, W., J. Hensen, M. Wiederholt. 1997 Graefes Arch. Clin. Exp. Ophthalmol. 235, 551-552.

8. Ringvold, A. 1966.- Exp. Eye Res.62, 261-264.

9. Kuchle, M., T.Ho. 1994 - Invest. Ophthalmol. Vis. Sci. 35, 748-752.

10. Kuchle, M., N. Nguyen. 1995 - Ophthalmic Res. 27,Supll. 1, 136-142.

11. Gospodarovitz, D. 1975 - J. Biol. Chem. 250, 2515-2520.

12. James, R., R. Bradshaw. 1984 - Ann. Rev. Biochem. 53, 259-292.

13. Tripathi, R., N. Borisuth, J.Li. 1994 - J.Glaucoma, 3,248-258.

14. Prince, A., B. Streeten, R. Ritch. 1987 - Arch. Ophthalmol.,105, 1076-1082.

15. Ramachandran, E., R. Frank, A, Kennedy. 1993 - Invest. Ophthalmol. Vis. Sci. 34, 586-589.

16. Shichi, H., T. Page, M. Sachuri, D. Shin. 1997 - J.Ocul. Pharmacol. Ther. 13, 201-206.

17. Triantos, A., G. Koliakos, E. Kavoukopoulos. 1995 - Biochem. Mol. Biol. Intern. 37,737-745.

18. Jampel, H., J. Moon, H. Quigley, Y. Barron. 1998 - Arch. Ophthalmol.116, 281-285. 